FACTA UNIVERSITATIS (NIŠ)

Ser. Math. Inform. Vol. 35, No 2 (2020), 459-469

https://doi.org/10.22190/FUMI2002459N

\title{
I-LOCALIZED SEQUENCES IN METRIC SPACES
}

\author{
Anar Adiloğlu Nabiev, Ekrem Savaş and Mehmet Gürdal
}

(C) 2020 by University of Niš, Serbia | Creative Commons Licence: CC BY-NC-ND

\begin{abstract}
In this paper, we have introduced $\mathcal{I}$-localized and $\mathcal{I}^{*}$-localized sequences in metric spaces and investigated some basics properties of the $\mathcal{I}$-localized sequences related with $\mathcal{I}$-Cauchy sequences. Also, we have obtained some necessary and sufficient conditions for the $\mathcal{I}$-localized sequences to be an $\mathcal{I}$-Cauchy sequences. We have also defined uniformly $\mathcal{I}$-localized sequences on metric spaces and its relation with $\mathcal{I}$-Cauchy sequences have been obtained.
\end{abstract}

Keywords: $\mathcal{I}$-Cauchy sequences; $\mathcal{I}$-localized sequences; $\mathcal{I}^{*}$-localized sequences.

\section{Introduction and preliminaries}

The localized sequences defined in [7] can be understood as a typical generalization of a Cauchy sequence in metric spaces. Using the properties of localized sequences and the locator of a sequence, some interesting results related to closure operators in metric spaces have been obtained in [7]. If $X$ is a metric space with a metric $d(\cdot, \cdot)$ and $\left(x_{n}\right)$ is a sequence of points in $X$, we call the sequence $\left(x_{n}\right)$ to be localized in some subset $M \subset X$ if the number sequence $\alpha_{n}=d\left(x_{n}, x\right)$ converges for all $x \in M$. The maximal subset on which $\left(x_{n}\right)$ is a localized sequence is called the locator of $\left(x_{n}\right)$. In addition, if $\left(x_{n}\right)$ is localized on $X$, then it becomes localized everywhere. If the locator of a sequence $\left(x_{n}\right)$ contains all elements of this sequence, except of a finite number of elements, then $\left(x_{n}\right)$ is called localized in itself. For the above notations and further properties of the localized sequences we refer to [7]. It is important to remark that, every Cauchy sequence in $X$ is localized everywhere. It is also an interesting fact that if $A: X \rightarrow X$ is a mapping with the condition $d(A x, A y) \leq d(x, y)$ for all $x, y \in X$, then for every $x \in X$ the sequence $\left(A^{n} x\right)$ is localized at every fixed point of the mapping $A$. This means that fixed points of the mapping $A$ is contained in the locator of the sequence $\left(A^{n} x\right)$. Motivating the above-mentioned facts the authors of the present study have recently introduced

Received March 21, 2019; accepted May 27, 2019

2010 Mathematics Subject Classification. Primary 40A35 
the notations of a statistically localized sequence and the statistically locator of a sequence [9] where important properties of statistically localized sequences have been investigated.

In the present paper, the main purpose is to generalize the concept of statistically localized sequence using the notation of ideal $\mathcal{I}$ of subset of the set $\mathbb{N}$ of positive integers. Note that the $\mathcal{I}$-convergence of sequences of real numbers and $\mathcal{I}$-convergence of sequences in metric spaces were also defined and investigated (see $[4,5])$.

Recall that for a non-empty set $X$, the family $\mathcal{I} \subset 2^{X}$ is an ideal if and only if for each $A, B \in \mathcal{I}$ we have $A \cup B \in \mathcal{I}$ and for each $A \in \mathcal{I}$ and $B \subset A$ we have $B \in \mathcal{I}$ (see [6]). Additionally, a non-empty family of set $\mathcal{F} \subset 2^{X}$ is a filter on $X$ if and only if $\varnothing \notin \mathcal{F}$, for each $A, B \in \mathcal{F}$ we have $A \cap B \in \mathcal{F}$ and for each $A \in \mathcal{F}$ and each $B \supset A$ we have $B \in \mathcal{F}$. In addition, an ideal $\mathcal{I}$ is called non-trivial if $\mathcal{I} \neq \varnothing$ and $X \notin \mathcal{I}$. Then, $\mathcal{I} \subset 2^{X}$ is a non-trivial ideal if and only if $\mathcal{F}=\mathcal{F}(\mathcal{I})=\{X \backslash A: A \in \mathcal{I}\}$ is a filter on $X$. A non-trivial ideal $\mathcal{I} \subset 2^{X}$ is called admissible if and only if $\mathcal{I} \supset\{\{x\}: x \in X\}($ see $[4,5])$.

Let $(X, d)$ be a fixed metric space and $\mathcal{I}$ denotes a non-trivial ideal of subsets of $\mathbb{N}$.

Definition 1.1. ([4]) A sequence $\left(x_{n}\right)_{n \in \mathbb{N}}$ of elements of $X$ is said to be $\mathcal{I}$-convergent to $\xi \in X$ and it will be denoted as $\mathcal{I}$ - $\lim _{n \rightarrow \infty} x_{n}=\xi$ if and only if

$$
A(\varepsilon)=\left\{n \in \mathbb{N}: d\left(x_{n}, \xi\right) \geq \varepsilon\right\} \in \mathcal{I}
$$

for any $\varepsilon>0$.

Definition 1.2. ([8]) A sequence $\left(x_{n}\right)_{n \in \mathbb{N}}$ of element of $X$ is said to be $\mathcal{I}$-Cauchy sequence if and only if there is $n_{0} \in N$ such that

$$
A(\varepsilon)=\left\{n \in \mathbb{N}: d\left(x_{n}, x_{n_{0}}\right) \geq \varepsilon\right\} \in \mathcal{I} \text { for each } \varepsilon>0 .
$$

Note that the notations of $\mathcal{I}^{*}$-convergent and $\mathcal{I}^{*}$-Cauchy sequences are also related to $\mathcal{I}$-convergence.

Definition 1.3. ([4]) A sequence $\left(x_{n}\right)_{n \in \mathbb{N}}$ of elements of $X$ is said to be $\mathcal{I}^{*}$ convergent to $\xi \in X$ if and only if there exists a set $M \in \mathcal{F}(\mathcal{I})$ such that $\lim _{k \rightarrow \infty} d\left(x_{m_{k}}, \xi\right)=$ 0 and $M=\left\{m_{1}<m_{2}<\ldots<m_{k}<\ldots\right\} \subset \mathbb{N}$.

Definition 1.4. ([8]) A sequence $\left(x_{n}\right)$ of elements of $X$ is said to be $\mathcal{I}^{*}$-Cauchy sequence if and only if there is a set $M=\left\{m_{1}<m_{2}<\ldots<m_{k}\right\}$ such that

$$
\lim _{k, p \rightarrow \infty} d\left(x_{m_{k}}, x_{m_{p}}\right)=0 .
$$


Note that $\mathcal{I}^{*}$-convergent and $\mathcal{I}^{*}$-Cauchy sequences imply $\mathcal{I}$-convergent and $\mathcal{I}$ Cauchy sequences, respectively. Moreover, if $\mathcal{I}$ is an ideal with property $(A P)$ (see [4]), then $\mathcal{I}$ and $\mathcal{I}^{*}$-convergence coincide. Also, in this case $\mathcal{I}$ and $\mathcal{I}^{*}$-Cauchy sequences are the same (see [8]). More property and fact about ideal convergence and statistical convergence are contained, for instance, in Gürdal et al. [2], Nuray et al. [10], Savaş and Gürdal [11, 12], Şahiner et al. [13] and Yegül and Dündar [14].

In this paper, we have defined $\mathcal{I}$-localized and $\mathcal{I}^{*}$-localized sequences in metric spaces and investigated some basics properties of $\mathcal{I}$-localized sequences related with $\mathcal{I}$-Cauchy sequences.

\section{2. $\quad \mathcal{I}$ and $\mathcal{I}^{*}$-localized sequences}

Let $(X, d)$ is a metric space and $\mathcal{I}$ is a non-trivial ideal of subsets of $\mathbb{N}$.

Definition 2.1. (a) A sequence $\left(x_{n}\right)_{n \in \mathbb{N}}$ of elements of $X$ is said to be $\mathcal{I}$-localized in the subset $M \subset X$ if and only if for each $x \in M, \mathcal{I}-\lim _{n \rightarrow \infty} d\left(x_{n}, x\right)$ exists, i.e. the real number sequence $\alpha_{n}=d\left(x_{n}, x\right)$ is $\mathcal{I}$-convergent.

(b) the maximal set on which a sequence $\left(x_{n}\right)$ is $\mathcal{I}$-localized we call the $\mathcal{I}$-locator of $\left(x_{n}\right)$ and we denote this set as $\operatorname{loc}_{\mathcal{I}}\left(x_{n}\right)$.

(c) A sequence $\left(x_{n}\right)$ is called $\mathcal{I}$-localized everywhere if $\mathcal{I}$-locator of $\left(x_{n}\right)$ coincides with $X$, i.e. $\operatorname{loc}_{\mathcal{I}}\left(x_{n}\right)=X$.

$(d)$ A sequence $\left(x_{n}\right)$ is called $\mathcal{I}$-localized in itself if

$$
\left\{n \in \mathbb{N}: x_{n} \notin l o c_{\mathcal{I}}\left(x_{n}\right)\right\} \subset \mathcal{I} .
$$

From this definition, we immediately have that if $\left(x_{n}\right)$ is an $\mathcal{I}$-Cauchy sequence then, it is $\mathcal{I}$-localized everywhere. Indeed, since

$$
\left|d\left(x_{n}, x\right)-d\left(x_{n_{0}}, x\right)\right| \leqslant d\left(x_{n}, x_{n_{0}}\right)
$$

we have

$$
\left\{n \in \mathbb{N}:\left|d\left(x_{n}, x\right)-d\left(x_{n_{0}}, x\right)\right| \geqslant \varepsilon\right\} \subset\left\{n \in \mathbb{N}: d\left(x_{n}, x_{n_{0}}\right) \geqslant \varepsilon\right\}
$$

which indicates that the sequence is $\mathcal{I}$-localized if it is $\mathcal{I}$-Cauchy sequence.

We also have that, every $\mathcal{I}$-convergent sequence is $\mathcal{I}$-localized. Note that, if $\mathcal{I}$ is an admissible ideal then

(i) every localized sequence in $X$ is $\mathcal{I}$-localized sequence in $X$.

(ii) if additionally $X$ is a vector space, then the sum of two $\mathcal{I}$-localized sequences is $\mathcal{I}$-localized and also multiplication of $\mathcal{I}$-localized sequence to a constant is also $\mathcal{I}$-localized.

Remark 2.1. If $\left(x_{n}\right)$ and $\left(y_{n}\right)$ are two $\mathcal{I}$-localized number sequences, then $\left(x_{n} y_{n}\right)$, $\left(\frac{x_{n}}{y_{n}}\right), y_{n} \neq 0$, are also $\mathcal{I}$-localized sequences. 
$\mathcal{I}^{*}$-localized sequences could also be defined in metric spaces. Then we call the sequence $\left(x_{n}\right)$ to be $\mathcal{I}^{*}$-localized in a metric space $X$ if and only if the number sequence $d\left(x_{n}, x\right)$ is $\mathcal{I}^{*}$-convergent for each $x \in X$. Therefore, every $\mathcal{I}^{*}$-convergent or $\mathcal{I}^{*}$-Cauchy sequence in a metric space $X$ is $\mathcal{I}^{*}$-localized in $X$.

It is known that for admissible ideal $\mathcal{I}^{*}$-Cauchy criteria and $\mathcal{I}^{*}$-convergence, implies that $\mathcal{I}$-Cauchy criteria and $\mathcal{I}$-convergence, respectively. Moreover, for the admissible ideal with the property $(A P)$ the notions of $\mathcal{I}$ and $\mathcal{I}^{*}$-Cauchy sequences; $\mathcal{I}$-convergent and $\mathcal{I}^{*}$-convergent sequences coincide.

Lemma 2.1. Let $\mathcal{I}$ is an admissible ideal on $\mathbb{N}$ and $X$ is a metric space. If a sequence $\left(x_{n}\right) \subset X$ is $\mathcal{I}^{*}$-localized on the set $M \subset X$, then $\left(x_{n}\right)$ is $\mathcal{I}$-localized on the set $M$ and $\operatorname{loc}_{\mathcal{I}_{*}}\left(x_{n}\right) \subset \operatorname{loc}_{\mathcal{I}}\left(x_{n}\right)$.

Proof. If $\left(x_{n}\right)$ is $\mathcal{I}^{*}$-localized on $M$, then there exist a set $H \in \mathcal{I}$ such that for $H^{C}=\mathbb{N} \backslash H=\left\{k_{1}<k_{2}<\ldots<k_{j}\right\}$ we have

$$
\lim _{j \rightarrow \infty} d\left(x_{j}, x\right)
$$

for each $x \in M$. Then, the sequence $d\left(x_{n}, x\right)$ is an $\mathcal{I}^{*}$-Cauchy sequence which implies the $d\left(x_{n}, x\right)$ is an $\mathcal{I}$-Cauchy sequences (see [8]). Therefore; the number sequence $d\left(x_{n}, x\right)$ is $\mathcal{I}$-convergent, i.e. $\left(x_{n}\right)$ is $\mathcal{I}$-localized on the set $M$.

Lemma 2.2. Suppose $(X, d)$ is a metric space, then

(i) if $X$ has no limit point, then $\mathcal{I}$ and $\mathcal{I}^{*}$-localized sequences are the same in $X$ and $\mathcal{I}_{l o c}\left(x_{n}\right)=\mathcal{I}_{l o c}^{*}\left(x_{n}\right)$ for any $\left(x_{n}\right) \in X$.

(ii) if $X$ has a limit point $\xi$, then there is an admissible ideal $\mathcal{I}$ for which there exists an $\mathcal{I}$-localized sequence $\left(y_{n}\right) \subset X$ such that $\left(y_{n}\right)$ is not $\mathcal{I}^{*}$-localized.

Proof. (i) If $X$ has no any limit point, then the notions $\mathcal{I}$ and $\mathcal{I}^{*}$-convergence coincide in $X$ (see [4]). Therefore, if $\left(x_{n}\right)$ is $\mathcal{I}$-localized then it is $\mathcal{I}^{*}$-localized also and by the Lemma 2.1 , we have $\mathcal{I}_{l o c}\left(x_{n}\right)=\mathcal{I}_{l o c}^{*}\left(x_{n}\right)$.

(ii) Let $\xi$ is a limit point of $X$. Then there exists a sequence $\left(x_{n}\right)$ such that $\lim _{n \rightarrow \infty} d\left(x_{n}, \xi\right)=0$. Let $D=\bigcup_{j=1}^{\infty} D_{j}, D_{j}=\left\{\alpha^{j-1}(2 s-1): s \in \mathbb{N}\right\}(j=1,2, \ldots)$ is a decomposition of integers and $\mathcal{E}$ is an ideal of sets $A \subset \mathbb{N}$ such that each $A$ intersects only a finite member of $D_{j}$ (see [4]). Let us define the sequence $y_{n}=x_{j}$ for $n \in D_{j}$. Then, from Theorem 3.1 in [4], we have that the sequence $\left(y_{n}\right)$ is $\mathcal{I}$ localized in $X$. This means that for each $x \in X$ we have $\mathcal{I}-\lim _{n \rightarrow \infty} d\left(y_{n}, x\right)=\alpha(x)$. Let us define $d\left(x_{n}, x\right)=\alpha_{n}(x)$. It is easy to show that $\mathcal{I}-\lim _{n \rightarrow \infty} \alpha_{n}(x)=\alpha(x)$. If for some $x \in X$ we have $\mathcal{I}^{*}-\lim _{n \rightarrow \infty} \alpha_{n}(x)=\alpha(x)$, then there is $H \in \mathcal{E}$ such that for $M=\left\{m_{1}<m_{2}<\ldots<m_{k}<\ldots\right\}=\mathbb{N} \backslash H$ we have $\lim _{k \rightarrow \infty} \alpha_{m_{k}}(x)=\alpha(x)$. According to the definition of $\mathcal{E}$, the integer $\ell \in \mathbb{N}$ such that $H \subset \Delta_{1} \cup \ldots \cup \Delta_{\ell}$ could be found yet, then $\Delta_{\ell+1} \subset \mathbb{N} \backslash H=M$. Therefore; for many infinite $k^{\prime}$ s, we have $\alpha_{m_{k}}(x) \rightarrow \alpha_{\ell+1}(x)$. This contradicts that $\alpha_{m_{k}}(x) \rightarrow \alpha(x)$. Hence, the sequence $\left(y_{n}\right)$ is $\mathcal{I}$-localized but not $\mathcal{I}^{*}$-localized. This proves Lemma 2.2 . 
We recall that a necessary and sufficient condition of equivalency of $\mathcal{I}$ and $\mathcal{I}^{*}$ convergent, also $\mathcal{I}$ and $\mathcal{I}^{*}$-Cauchy condition, is so called $(A P)$ properties of an admissible ideal $\mathcal{I} \subset 2^{\mathbb{N}}$ (see [4]) which means that for every family $\left\{A_{i}\right\}_{i \in \mathbb{N}}$ with $A_{i} \cap A_{j}=\varnothing(i \neq j), A_{i} \in \mathcal{I}(i \in \mathbb{N})$, there is a family $\left\{B_{i}\right\}_{i \in \mathbb{N}}$ such that $\left(A_{j} \backslash B_{i}\right) \cup$

$\left(B_{j} \backslash A_{j}\right)$ for all $j \in \mathbb{N}$ and a limit set $B=\bigcup_{j=1}^{\infty} B_{j} \in \mathcal{I}$. From the Theorem 3.2 in [4], if $\mathcal{I}$ is an admissible ideal with the property $(A P)$ and $\left(x_{n}\right)$ is $\mathcal{I}$-localized sequence, then $\mathcal{I}^{*}-\lim _{n \rightarrow \infty} d\left(x_{m}, x\right)$ exists for each $x$. This means that, for any admissible ideal with the property $(A P) \mathcal{I}$-localized sequence is also $\mathcal{I}^{*}$-localized. In contrast, if every $\mathcal{I}$-localized sequence is also $\mathcal{I}^{*}$-localized and the metric space $(X, d)$ has at least one limit point, then $\mathcal{I}$ has the property $(A P)$. Indeed, if $\xi$ is a limit point of $X$ then there exists a sequence $\left(x_{n}\right)$ such that $d\left(x_{n}, \xi\right)=0$. Then, putting $y_{n}=x_{j}$ for $n \in A_{j}$, where $\left\{A_{n}\right\}_{n \in \mathbb{N}}$ is a disjoint family of non-empty sets from $\mathcal{I}$, we have $\mathcal{I}$ - $\lim _{n \rightarrow \infty} y_{n}=\xi$. Hence, $\left(y_{n}\right)$ is $\mathcal{I}$-localized sequence. By the assumption of $\left(y_{n}\right)$ is $\mathcal{I}^{*}$-localized, $\mathcal{I}^{*}-\lim _{n \rightarrow \infty} d\left(y_{n}, x\right)$ exists for each $x$. So, there is a set $B(x) \in \mathcal{I}$ such that if $M(x)=\mathbb{N} \backslash B(x)=\left\{m_{1}(x)<m_{2}(x)<\ldots\right\}$, then the limit

$$
\lim _{k \rightarrow \infty} d\left(y_{m_{k}}, x\right)=\alpha(x)
$$

exist. In addition, for each fixed $x$ it could be proved that $\mathcal{I}$ has the property $(A P)$ as in [4].

The investigation of other properties of the $\mathcal{I}$-localized sequences have been given in the following section.

\section{Basic properties of ideal localized sequences}

Proposition 3.1. Every $\mathcal{I}$-localized sequence is $\mathcal{I}$-bounded.

Proof. Let $\left(x_{n}\right)$ is $\mathcal{I}$-localized. Then, the number sequence $d\left(x_{n}, x\right)$ is $\mathcal{I}$-convergent for some $x \in X$. This means that $\left\{n \in \mathbb{N}: d\left(x_{n}, x\right)>K\right\} \in \mathcal{I}$ for some $K>0$. Consequently, the sequence $\left(x_{n}\right)$ is $\mathcal{I}$-bounded.

Proposition 3.2. Let $\mathcal{I}$ is an admissible ideal with the property $(A P)$ and $L=$ $\operatorname{loc}_{\mathcal{I}}\left(x_{n}\right)$. Also, a point $z \in X$ be such that for any $\varepsilon>0$ there exists $x \in L$ satisfying

$$
\left\{n \in \mathbb{N}:\left|d\left(x, x_{n}\right)-d\left(z, x_{n}\right)\right| \geqslant \varepsilon\right\} \in \mathcal{I} .
$$

Then $z \in L$.

Proof. It is enough to show that the number sequence $\alpha_{n}=d\left(x_{n}, z\right)$ is an $\mathcal{I}$-Cauchy sequence. Let $\varepsilon>0$ and $x \in L=\operatorname{loc}_{\mathcal{I}}\left(x_{n}\right)$ is a point with the property (1). By adopting the $(A P)$ property of $\mathcal{I}$, we have

$$
\left|d\left(x, x_{k_{n}}\right)-d\left(z, x_{k_{n}}\right)\right| \rightarrow 0 \text { as } n \rightarrow \infty,
$$


and

$$
\left|d\left(x_{k_{n}}, x\right)-d\left(x_{k_{m}}, x\right)\right| \rightarrow 0 \text { as } m, n \rightarrow \infty
$$

where $K=\left\{k_{1}<k_{2}<\ldots<k_{n}<\ldots\right\} \in \mathcal{F}(\mathcal{I})$. Hence for any $\varepsilon>0$ there is $n_{0} \in \mathbb{N}$ such that

$$
\left|d\left(x, x_{k_{n}}\right)-d\left(z, x_{k_{n}}\right)\right|<\frac{\varepsilon}{3}
$$

and

$$
\left|d\left(x, x_{k_{n}}\right)-d\left(x, x_{k_{m}}\right)\right|<\frac{\varepsilon}{3}
$$

for all $n \geq n_{0}, m \geq m_{0}$.

Now, combining (2) and (3) together with the following estimation

$$
\begin{gathered}
\left|d\left(z, x_{k_{n}}\right)-d\left(z, x_{k_{m}}\right)\right| \\
\leq\left|d\left(z, x_{k_{n}}\right)-d\left(x, x_{k_{n}}\right)\right|+\left|d\left(x, x_{k_{n}}\right)-d\left(x, x_{k_{m}}\right)\right|+\left|d\left(x, x_{k_{m}}\right)-d\left(z, x_{k_{m}}\right)\right|
\end{gathered}
$$

we obtain

$$
\left|d\left(z, x_{k_{n}}\right)-d\left(z, x_{k_{m}}\right)\right|<\varepsilon
$$

for all $n \geq n_{0}, m \geq n_{0}$, which gives

$$
\left|d\left(z, x_{k_{n}}\right)-d\left(z, x_{k_{m}}\right)\right| \rightarrow 0 \text { as } m, n \rightarrow \infty
$$

for $K=\left(k_{n}\right) \subset N$ and $K \in \mathcal{F}(\mathcal{I})$. This result implies that $d\left(x_{n}, z\right)$ is an $\mathcal{I}$-Cauchy sequence which finalizes the proof.

Proposition 3.3. The $\mathcal{I}$-locator of any sequence is a closed subset of the metric space $X$.

Proof. Let $z \in \overline{l o c}_{\mathcal{I}}\left(x_{n}\right)$. Then, for any $\varepsilon>0$ the ball $B(z, \varepsilon)$ will contain a point $x \in \operatorname{loc}_{\mathcal{I}}\left(x_{n}\right)$. Therefore;

$$
\left\{n \in \mathbb{N}:\left|d\left(x, x_{n}\right)-d\left(z, x_{n}\right)\right| \geqslant \varepsilon\right\} \in \mathcal{I}
$$

for any $\varepsilon>0$, since for each $n \in \mathbb{N}$

$$
\left|d\left(x, x_{n}\right)-d\left(z, x_{n}\right)\right| \leq d(z, x)<\varepsilon .
$$

Consequently, the hypothesis of Proposition 3.2 is satisfied. Then $z \in \operatorname{loc}_{\mathcal{I}}\left(x_{n}\right)$, i.e. $\operatorname{loc}_{\mathcal{I}}\left(x_{n}\right)$ is closed.

Recall that the point $z$ is an $\mathcal{I}$-limit point of the sequence $\left(x_{n}\right) \in X$ if there is a set

$$
K=\left\{k_{1}<k_{2}<\ldots<k_{n}\right\} \subset \mathbb{N}
$$


such that $K \notin \mathcal{I}$ and $\lim _{k \rightarrow \infty} x_{m_{k}}=z$. A point $\xi$ is said to be an $\mathcal{I}$-cluster point of the sequence $\left(x_{n}\right)$ if for each $\varepsilon>0$

$$
\left\{n \in \mathbb{N}: d\left(x_{n}, \xi\right)<\varepsilon\right\} \notin \mathcal{I}
$$

(see $[4,5])$. In addition, if $K=\left\{k_{1}<k_{2}<\ldots\right\} \in \mathcal{I}$, then the subsequence $\left(x_{k_{n}}\right)$ of the sequence $\left(x_{n}\right)$ is called $\mathcal{I}$-thin subsequence of the sequence $\left(x_{n}\right)$. If $M=$ $\left\{m_{1}<m_{2}<\ldots\right\} \notin \mathcal{I}$, then the sequence $x_{M}=\left(x_{m}\right)$ is called $\mathcal{I}$-nonthin subsequence of $\left(x_{n}\right)$.

Since $\left|d\left(x_{n}, y\right)-d(z, y)\right| \leq d\left(x_{n}, z\right)$, the following propositions could be given.

Proposition 3.4. If $z \in X$ is an $\mathcal{I}$-limit point (an $\mathcal{I}$-cluster point) of a sequence $\left(x_{n}\right) \in X$, then for each $y \in X$ the number $d(z, y)$ is an $\mathcal{I}$-limit point (an $\mathcal{I}$-cluster point) of the sequence $\left\{d\left(x_{n}, y\right)\right\}$.

Proposition 3.5. All $\mathcal{I}$-limit points (I-cluster points) of the $\mathcal{I}$-localized sequence $\left(x_{n}\right)$ have the same distance from each point $x$ of the locator $\operatorname{loc}_{\mathcal{I}}\left(x_{n}\right)$.

Proof. If $z_{1}$ and $z_{2}$ are two $\mathcal{I}$-limit points of the sequence $\left(x_{n}\right)$, then the numbers $d\left(z_{1}, x\right)$ and $d\left(z_{2}, x\right)$ are $\mathcal{I}$-limit points of the $\mathcal{I}$-convergent sequence $d\left(x, x_{n}\right)$. Consequently, $d\left(z_{1}, x\right)=d\left(z_{2}, x\right)$.

Proposition 3.6. $\quad \operatorname{loc}_{\mathcal{I}}\left(x_{n}\right)$ does not contain more than one $\mathcal{I}$-limit (I-cluster) point of the sequence $\left(x_{n}\right)$. Particularly, everywhere the localized sequence has not more than one $\mathcal{I}$-limit $(\mathcal{I}$-cluster) point.

Proof. If $x, y \in \operatorname{loc}_{\mathcal{I}}\left(x_{n}\right)$ are two $\mathcal{I}$-limit points of the sequence $\left(x_{n}\right)$, then by the Proposition 3.5, $d(x, x)=d(x, y)$. But $d(x, x)=0$. This implies that, $d(x, y)=0$ for $x \neq y$ which is a well-known contradiction.

Proposition 3.7. If the sequence $\left(x_{n}\right)$ has an $\mathcal{I}$-limit point $z \in \operatorname{loc}_{\mathcal{I}}\left(x_{n}\right)$, then $\mathcal{I}-\lim _{n \rightarrow \infty} x_{n}=z$.

Proof. The sequence $\left\{d\left(x_{n}, z\right)\right\}$ is $\mathcal{I}$-convergent and some $\mathcal{I}$-nonthin subsequence of this sequence converges to zero. Then, $\left(x_{n}\right)$ is $\mathcal{I}$-convergent to $z$.

Definition 3.1. For the given $\mathcal{I}$-localized sequence $\left(x_{n}\right)$, with the $\mathcal{I}$-locator $L=$ $\operatorname{loc}_{\mathcal{I}}\left(x_{n}\right)$, the number

$$
\sigma=\inf _{x \in L}\left(\mathcal{I}-\lim _{n \rightarrow \infty} d\left(x, x_{n}\right)\right)
$$

is called the $\mathcal{I}$-barrier of $\left(x_{n}\right)$.

Theorem 3.1. Let $\mathcal{I} \subset 2^{\mathbb{N}}$ is an ideal with $(A P)$ property. Then, an $\mathcal{I}$-localized sequence is $\mathcal{I}$-Cauchy sequence if and only if $\sigma=0$. 
Proof. Let $\left(x_{n}\right)$ is an $\mathcal{I}$-Cauchy sequence in a metric space $X$. Then, there is a set $K=\left\{k_{1}<k_{2}<\ldots<k_{n}\right\} \subset \mathbb{N}$ such that $K \in \mathcal{F}(\mathcal{I})$ and $\lim _{n, m \rightarrow \infty} d\left(x_{k_{n}}, x_{k_{m}}\right)=0$. Consequently, for each $\varepsilon>0$ there exists $n_{0} \in \mathbb{N}$ such that

$$
d\left(x_{k_{n}}, x_{k_{n_{0}}}\right)<\varepsilon \text { for all } n \geq n_{0} .
$$

Since $\left(x_{n}\right)$ is an $\mathcal{I}$-localized sequence, $\mathcal{I}$ - $\lim _{n \rightarrow \infty} d\left(x_{k_{n}}, x_{k_{n_{0}}}\right)$ exist and we get $\mathcal{I}$ $\lim _{n \rightarrow \infty} d\left(x_{k_{n}}, x_{k_{n_{0}}}\right) \leq \varepsilon$. Hence, $\sigma \leq \varepsilon$. Because $\varepsilon>0$, we also get $\sigma=0$.

Let now assume the converse by taking $\sigma=0$. Then, for each $\varepsilon>0$ there is an $x \in \operatorname{loc}_{\mathcal{I}}\left(x_{n}\right)$ such that $d(x)=\mathcal{I}$ - $\lim _{n \rightarrow \infty} d\left(x, x_{n}\right)<\frac{\varepsilon}{2}$. In this case

$$
\left\{n \in \mathbb{N}:\left|d(x)-d\left(x, x_{n}\right)\right| \geq \frac{\varepsilon}{2}-d(x)\right\} \in \mathcal{I}
$$

which implies $\left\{n \in \mathbb{N}: d\left(x, x_{n}\right) \geq \frac{\varepsilon}{2}\right\} \in \mathcal{I}$. Therefore, $\mathcal{I}$ - $\lim _{n \rightarrow \infty} d\left(x, x_{n}\right)=0$, i.e. $\left(x_{n}\right)$ is an $\mathcal{I}$-Cauchy sequence.

Remark 3.1. We have previously acquired from the proof of Theorem 3.1 that, if $\sigma=0$ then an ideal $\mathcal{I}$ will not have $(A P)$ properties. In other words, if an $\mathcal{I}$-barrier of a localized sequence is equal to zero, then it is an $\mathcal{I}$-Cauchy sequence.

Theorem 3.2. If the sequence $\left(x_{n}\right)$ is $\mathcal{I}$-localized in itself and $\left(x_{n}\right)$ contains an $\mathcal{I}$-nonthin Cauchy subsequence, then $\left(x_{n}\right)$ will be an $\mathcal{I}$-Cauchy sequence itself.

Proof. Let $\left(y_{n}\right)$ is an $\mathcal{I}$-nonthin Cauchy subsequence. It could be assumed that all members of $\left(y_{n}\right)$ belong to the $\operatorname{loc}_{\mathcal{I}}\left(x_{n}\right)$. Since $\left(y_{n}\right)$ is a Cauchy sequence, by Theorem 3.1, $\inf _{y_{n}} \lim _{m \rightarrow \infty} d\left(y_{m}, y_{n}\right)=0$. On the other hand, since $\left(x_{n}\right)$ is $\mathcal{I}$ localized in itself then,

$$
\text { I- } \lim _{m \rightarrow \infty} d\left(x_{m}, y_{n}\right)=\mathcal{I}-\lim _{m \rightarrow \infty} d\left(y_{m}, y_{n}\right)=0 .
$$

This implies that, the $\mathcal{I}$-barrier of $\left(x_{n}\right)$ is equal to zero: $\sigma=0$. Then, by using the Remark 3.1, we figure out that $\left(x_{n}\right)$ is an $\mathcal{I}$-Cauchy sequence.

Let $a \in X, r>0$ and $\mathcal{I} \subset 2^{\mathbb{N}}$ is an admissible ideal. Recall that the sequence $\left(x_{n}\right)$ in a metric space $(X, d)$ is called $\mathcal{I}$-bounded if there is a subset $K=$ $\left\{k_{1}<k_{2}<\ldots<k_{n} \subset \ldots\right\} \subset \mathbb{N}$ such that $K \in \mathcal{F}(\mathcal{I})$ and $\left(x_{k_{n}}\right) \subset B(a, r)$, where $B(a, r)$ is the ball with center at the point $a$ and radius $r$. Clearly, $\left(x_{k_{n}}\right)$ is a bounded sequence in $X$ sequence and it has a localized in itself subsequence (see [7]). As a result, the following assertion also becomes true.

Proposition 3.8. Every $\mathcal{I}$-bounded sequence in a metric space has an $\mathcal{I}$-localized in itself subsequence.

Theorem 3.3. If every $\mathcal{I}$-localized in itself sequence of $X$ is an $\mathcal{I}$-Cauchy sequence, then every bounded set in $X$ is totally bounded. 
Proof. Let $\left(x_{n}\right)$ is an $\mathcal{I}$-localized in itself sequence of metric space $(X, d)$, but the assertion is not true. Then, there is a bounded subset $M \subset X$ such that $d\left(x_{n}, x_{m}\right)>$ $\varepsilon(n \neq m)$ for some $\varepsilon>0$ and for some sequence $\left(x_{n}\right) \subset M$. Since $\left(x_{n}\right)$ is bounded by Proposition $3.8,\left(x_{n}\right)$ has an $\mathcal{I}$-localized in itself sequence $\left(x_{n}^{\prime}\right)$. Since $d\left(x_{n}^{\prime}, x_{m}^{\prime}\right)>\varepsilon$ for any $n \neq m$, the subsequence is not an Cauchy sequence. This contradicts our assumption.

Definition 3.2. We indicate an infinite subset $M \subset X$ is thick relatively to a nonempty subset $Y \subset X$ if for each $\varepsilon>0$ there is the $a$ point $y \in Y$ such that the ball $B(y, \varepsilon)$ has infinitely many points of $M$. In particular, if the set $M$ is thick relatively to its subset $Y \subset M$ then $M$ is called thick in itself.

Proposition 3.9. If every bounded infinite set of $X$ is thick in itself, then every $\mathcal{I}$-localized in itself sequence of $X$ is an $\mathcal{I}$-Cauchy sequence.

Proof. Let the assumption is satisfied and $\left(x_{n}\right)$ is an $\mathcal{I}$-localized in itself sequence of $X$. Then, $\left(x_{n}\right)$ is an $\mathcal{I}$-bounded sequence in $X$. This implies that there is an infinite set $M$ of points of $\left(x_{n}\right)$ such that $M$ is a bounded subset of $X$. By this assumption, $M$ is thick in itself. Then, for every $\varepsilon>0$ we can choose $x_{k} \in M$ such that the ball $B\left(x_{k}, \varepsilon\right)$ contains infinitely many points of $x_{1}^{\prime}, \ldots, x_{n}^{\prime}, \ldots$ of $X$. For the sequence $\left(x_{n}^{\prime}\right)$ the sequence $d\left(x_{n}^{\prime}, x_{k}\right)_{n=1}^{\infty}$ is $\mathcal{I}$-convergent and

$$
\mathcal{I}-\lim _{n \rightarrow n_{0}} d\left(x_{n}^{\prime}, x_{K}\right) \leq \varepsilon .
$$

Hence, the $\mathcal{I}$-barrier of $\left(x_{n}\right)$ is equal to zero so $\left(x_{n}\right)$ is an $\mathcal{I}$-Cauchy sequence.

Since every bounded subset of $X$ is totally bounded if and only if every bounded infinite subset of $X$ is thick in itself (see [7]), the equality of Theorem 3.3 and Proposition 3.9 have been figure out. Moreover, from Theorem 3.2 and Theorem 3.1 it has been figured out that in Theorem 3.3 the assumption could be replaced equivalently with the assumption regarding every $\mathcal{I}$-bounded sequence to have an $\mathcal{I}$-Cauchy subsequence.

Proposition 3.10. If every $\mathcal{I}$-bounded sequence has an $\mathcal{I}$-Cauchy subsequence, then every bounded subset in $X$ is totally bounded.

Note that in separable metric spaces, Proposition 3.10 can be weakened.

Proposition 3.11. If every $\mathcal{I}$-bounded sequence of separable metric space $X$ has an $\mathcal{I}$-localized subsequence in everywhere, then every bounded subset of $X$ could be said that totally bounded.

Definition 3.3. A sequence $\left(x_{n}\right)$ in metric space $(X, d)$ is called uniformly $\mathcal{I}$ localized on a subset $M \subset X$ if the sequence $\left\{d\left(x, x_{n}\right)\right\}$ is uniformly $\mathcal{I}$-convergent for all $x \in M$. 
The following proposition is proved analogously to Proposition 3.2.

Proposition 3.12. Let sequence $\left(x_{n}\right)$ be uniformly $\mathcal{I}$-localized on the set $M \subset X$ and $z \in Y$ is such that for every $\varepsilon>0$ there is $y \in M$ for which

$$
\left\{n \in \mathbb{N}:\left|d\left(z, x_{n}\right)-d\left(y, x_{n}\right)\right| \geqslant \varepsilon\right\} \in \mathcal{I}
$$

is satisfied. Then, $z \in \operatorname{loc}_{\mathcal{I}}\left(x_{n}\right)$ and $\left(x_{n}\right)$ is uniformly $\mathcal{I}$-localized on the set of such points $z$.

Remark 3.2. The function $d_{M}(x, z)=\sup _{y \in M}|d(x, y)-d(y, z)|, x, z \in Y$ is called a pseudo metric on the set $Y$.

It is easy to prove by standard techniques (see $[1,3]$ ) that a sequence $\left(x_{n}\right)$ is uniformly $\mathcal{I}$-localized on the set $M \subset X$ if and only if $\left(x_{n}\right)$ is an $\mathcal{I}$-Cauchy sequence with respect to the metric $d_{M}(x, z)$.

Theorem 3.4. Every uniformly $\mathcal{I}$-localized in itself sequence is $\mathcal{I}$-Cauchy sequence.

Proof. Let $\left\{n \in \mathbb{N}: x_{n} \in M\right\} \in \mathcal{F}(\mathcal{I})$ the uniformly $\mathcal{I}$-localized in itself sequence $\left(x_{n}\right)$. From the definition of $d_{M}$ we get that if at most one of the points $x, z$ belongs to $M$, then $d_{M}(x, z)=d(x, z)$. In particular, there is $n_{0} \in \mathbb{N}$ such that $\left\{n \in \mathbb{N}: d_{M}\left(x_{n}, x_{n_{0}}\right)=d\left(x_{n}, x_{n_{0}}\right)\right\} \in \mathcal{F}(\mathcal{I})$. Now the assertion is obtained from Proposition 3.2.

\section{Acknowledgment}

We would like to thank the referees for careful reading and fruitful suggestions.

\section{REF E R E N C E S}

1. H. Busemann: The geometry of geodesics, Dover Publications, New York, USA, 2005.

2. M. Gürdal, A. ŞAhiner AND I. AÇIK: Approximation theory in 2-Banach spaces, Nonlinear Anal., 71(5-6) (2009), 1654-1661.

3. J. L. Kelley: General Topology, Springer-Verlag New York, 1955.

4. P. Kostyrko, M. Macaj and T. Salat: I-Convergence, Real Anal. Exchang, 26 (2000), 669-686.

5. P. Kostyrko, M. MacaJ, T. Salat and M. Sleziak: $\mathcal{I}$-Convergence and Extremal I-Limit Points, Math. Slovaca, 55 (2005), 443-464.

6. C. Kuratowski: Topologie I, PWN Warszawa, 1958.

7. L. N. Krivonosov: Localized sequences in metric spaces, Izv. Vyssh. Uchebn. Zaved Mat., 4 (1974), 45-54. 
8. A. Nabiev, S. Pehlivan And M. Gürdal: On I-Cauchy sequences, Taiwanese J. Math., 11 (2007), 569-566.

9. A. A. NABIEv, E. SAVAŞ AND M. GÜRdAl: Statistically localized sequences in metric spaces, J. Appl. Anal. Comp., 9(2) (2019), 739-746.

10. F. Nuray, U. Ulusu AND E. Dündar: Lacunary statistical convergence of double sequences of sets, Soft Computing, 20(7) (2016), 2883-2888.

11. E. SAVAŞ AND M. GÜRDAL: Certain summability methods in intuitionistic fuzzy normed spaces, J. Intell. Fuzzy. Syst., 27(4) (2014), 1621-1629.

12. E. SAVAŞ AND M. Gürdal: Ideal Convergent Function Sequences in Random 2Normed Spaces, Filomat, 30(3) (2016), 557-567.

13. A. ŞAHINER, M. GüRDAL AND T. YIĞIT: Ideal convergence characterization of the completion of linear n-normed spaces, Comput. Math. Appl., 61(3) (2011), 683-689.

14. S. Yegül and E. Dündar: Statistical convergence of double sequences of functions and some properties in 2-normed spaces, Facta Univ. Ser. Math. Inform. 33(5) (2018), 705-719.

\author{
Anar Adiloğlu Nabiev \\ Süleyman Demirel University \\ Department of Computer Engineering \\ 32260 Isparta, Turkey \\ anaradiloglu@sdu.edu.tr \\ Ekrem Savaş \\ Uşak University \\ Department of Mathematics \\ 64000 Uşak, Turkey \\ ekremsavas@yahoo.com \\ Mehmet Gürdal \\ Süleyman Demirel University \\ Department of Mathematics \\ 32260 Isparta, Turkey \\ gurdalmehmet@sdu.edu.tr
}

